\title{
Stiffness of the Crystal-Liquid Interface in a Hard- Sphere Colloidal System Measured from Capillary Fluctuations
}

\section{Citation}

Ramsteiner, I. B., D. A. Weitz, and F. Spaepen. 2010. Stiffness of the Crystal-Liquid Interface in a Hard-Sphere Colloidal System Measured from Capillary Fluctuations. Physical Review E 82, no. 4: 041603.

\section{Published Version}

doi:10.1103/PhysRevE.82.041603

\section{Permanent link}

http://nrs.harvard.edu/urn-3:HUL.InstRepos:13041039

\section{Terms of Use}

This article was downloaded from Harvard University's DASH repository, and is made available under the terms and conditions applicable to Other Posted Material, as set forth at http:// nrs.harvard.edu/urn-3:HUL.InstRepos:dash.current.terms-of-use\#LAA

\section{Share Your Story}

The Harvard community has made this article openly available.

Please share how this access benefits you. Submit a story.

\section{Accessibility}




\title{
Stiffness of the crystal-liquid interface in a hard-sphere colloidal system measured from capillary fluctuations
}

\author{
I. B. Ramsteiner, ${ }^{1}$ D. A. Weitz, ${ }^{1,2}$ and F. Spaepen ${ }^{1}$ \\ ${ }^{1}$ School of Engineering and Applied Sciences, Harvard University, Cambridge, Massachusetts 02138, USA \\ ${ }^{2}$ Physics Department, Harvard University, Cambridge, Massachusetts 02138, USA
}

(Received 22 June 2010; published 20 October 2010)

\begin{abstract}
Face-centered cubic single crystals of $\sigma=1.55 \mu \mathrm{m}$ diameter hard-sphere silica colloidal particles were prepared by sedimentation onto (100) and (110) oriented templates. The crystals had a wide interface with the overlaying liquid that was parallel to the template. The location of the interface was determined by confocal microscopic location of the particles, followed by identification of the crystalline and liquid phases by a bond-orientation order parameter. Fluctuations in the height of the interface about its average position were recorded for several hundred configurations. The interfacial stiffness $\tilde{\gamma}$ was determined from the slope of the inverse squared Fourier components of the height profile vs the square of the wave number, according to the continuum capillary fluctuation method. The offset of the fit from the origin could quantitatively be accounted for by gravitational damping of the fluctuations. For the (100) interface, $\tilde{\gamma}=(1.3 \pm 0.3) k_{B} T / \sigma^{2}$; for the (110) interface, $\tilde{\gamma}=(1.0 \pm 0.2) k_{B} T / \sigma^{2}$. The interfacial stiffness of both interfaces was found to be isotropic in the plane. This is surprising for the (110), where crystallography predicts twofold symmetry. Sedimentation onto a (111) template yielded a randomly stacked hexagonal crystal with isotropic $\tilde{\gamma}=0.66 k_{B} T / \sigma^{2}$. This value, however, is less reliable than the two others due to imperfections in the crystal.
\end{abstract}

DOI: 10.1103/PhysRevE.82.041603

PACS number(s): 68.08.De, 68.37.-d, 82.70.Dd, 05.40.-a

\section{INTRODUCTION}

Interfaces between crystals and their own melt are of great interest in both materials science and statistical physics. They are technologically important because their properties govern the solidification process, which in turn determines the microstructure of a material. Of particular interest is the formation of dendrites, which results from the interplay between diffusion of heat and matter, capillary forces and crystalline anisotropy $[1,2]$. Dendrite growth is a prime example of a multiscale phenomenon: Since the crystal anisotropy originates on the atomic scale, the typical dendrite tip has a micron-size curvature, and the dendrite itself measures millimeters or centimeters, the length scale spans at least seven orders of magnitude. For this reason numerical simulations of dendritic growth are continuum models that use macroscopic parameters that are in turn obtained from atomic scale simulations. Current modeling uses the phase field method with input parameters the kinetic coefficient and interfacial free energy (IFE) derived from Monte Carlo (MC) or molecular dynamics (MD) simulations [3].

Extracting the IFE $\gamma(\theta)$ as a function of the crystal surface orientation $\theta$ from atomic scale simulation data is a challenging problem by itself. One reason is that storage and computing power limit the simulated volume to dimensions at which the discrete nature of the system may mask the continuum properties. At the same time very high precision is required, since dendritic growth of metals for example is believed to have its origin in subtle anisotropies $(\sim 1 \%)$ of $\gamma(\theta)$. Two methods for determining $\gamma(\theta)$ have been proposed: one is the so-called cleaving method (CM), introduced by Broughton and Gilmer, which involves reversibly splitting a simulated crystal along a specified crystal plane by gradually applying an external potential, "melting" one half of the cleaved system, bringing the parts together again, and calculating $\gamma$ from the total reversible work necessary for that procedure [4]. An alternative approach is the capillary fluctuation method (CFM), first applied to crystal-melt interfaces by Hoyt, Asta and Karma $[5,6]$. It analyzes the equilibrium fluctuations of a molecularly rough, but otherwise flat and stationary interface. With its surface normal in the $z$ direction, the interface at the time $t$ is characterized by a height profile $h(x, y, t)$. The mean amplitude of this function is determined by the competition between thermal fluctuations and the free energy cost of locally increasing the interface area and changing its orientation $\theta$. The latter adds a fundamental difference to the otherwise identical phenomenon at liquid-liquid or liquid-gas interfaces with an isotropic $\gamma$. Therefore, the CFM does not give direct access to $\gamma$, but to the interfacial stiffness

$$
\widetilde{\gamma}(\theta)=\gamma(\theta)+\frac{\partial^{2} \gamma(\theta)}{\partial \theta^{2}} .
$$

This is actually an advantage when investigating subtle anisotropies, since these are an order of magnitude stronger for $\tilde{\gamma}$ than for $\gamma$. The average squared Fourier amplitudes are related to $\tilde{\gamma}$ by

$$
\left\langle\left|h_{\mathbf{q}}\right|^{2}\right\rangle_{t}=\frac{k_{B} T}{\widetilde{\gamma}(\theta) q^{2}},
$$

where $k_{B} T$ is the Boltzmann term and $q$ in-plane wave vector. Angle brackets denote time averages throughout this paper, even if the index $t$ is omitted. While both approaches have been successfully employed in the past, the CM is considered to yield more precise absolute values for the interfacial free energy, while the CFM is superior in elucidating small anisotropies [7]. The CM is necessarily a method for numerical simulations, while the CFM could in principle be applied 
TABLE I. Interfacial stiffness of hard sphere crystal-melt interfaces obtained by the capillary fluctuation method from molecular dynamics and Monte Carlo simulations. Numerical values are given in units of $k_{B} T / \sigma^{2}$ (the accuracy in parentheses) where $\sigma$ is the particle diameter.

\begin{tabular}{ccccc}
\hline$(100)[001]$ & $(110)[\overline{1} 10]$ & $(110)[001]$ & $(111)[\overline{1} 10]$ & Ref. \\
\hline 0.55 & 0.49 & 0.71 & 0.80 & $\mathrm{MC}(2005)[29]$ \\
$0.44(3)$ & $0.42(3)$ & $0.70(3)$ & $0.67(4)$ & $\mathrm{MD}(2006)[7]$ \\
$0.425(10)$ & & $0.410(16)$ & $0.74(3)$ & $\mathrm{MD}(2006)[30]$ \\
\hline \hline
\end{tabular}

to experimental data. Unfortunately, such data is almost completely lacking.

Since the interface is buried between two dense phases, it is inaccessible to most techniques. Sophisticated methods have been devised to "freeze" equilibrium configurations for postsolidification analysis $[8,9]$, but the interface remains elusive to in situ experiments, particularly on the atomic level, where the length and time scales make any in situ experiments highly challenging.

In recent years, the advances in confocal microscopy have provided a powerful tool for experiments in biology and soft matter physics. The ability to track colloidal particles driven by Brownian motion and interacting via potentials that can be tuned in range and depth has opened up unprecedented opportunities to put concepts of statistical physics directly to the test [10-14]. Prasad et al. [15] have reviewed its use in the modeling of atomic or molecular systems. For example, capillary waves at colloidal fluid-gas interfaces have already been observed by Aarts et al. and Derks et al. [16-18] If the particles are not density-matched with the liquid, a vertical concentration gradient occurs that can be exploited to form a stationary equilibrium interface between the crystalline and fluid phases [19-22]. Density-matched dispersions would of course be closer to hard sphere computer simulations. But since a real colloidal system has no periodic boundary conditions to keep the interface straight and in place, curved interfaces at random angles would move in and out of focus within minutes. While first successes in imaging colloidal crystal-melt interfaces have been reported by Dullens et al. [23], fluctuations at such an interface have been observed very recently by Hernández-Guzmán and Weeks [24].

In this work we use colloids with the simplest interaction potential conceivable: hard spheres, which were also used in Refs. $[23,24]$. Despite a complete lack of attractive forces hard spheres form crystals from the liquid by a first order phase transition driven, counterintuitively, by entropy alone [25-27]. The only parameter in the athermal phase diagram is the packing fraction $\Phi$ : while the particles move freely around in a fluid-like fashion for $\Phi<0.494$, they form a face centered cubic (fcc) crystal for $\Phi>0.545$ by nucleation and growth [28].

Several numerical simulation studies have employed the CFM to measure the stiffness of the hard sphere crystal-melt interface $[7,29,30]$. The results are summarized in Table I. Although there are some discrepancies between the values for $\tilde{\gamma}(\theta)$, they have a similar order of magnitude. The values of the IFE $\gamma(\theta)$ obtained from these stiffnesses are also simi- lar in magnitude to those attained from simulation data by employing classical nucleation theory [31] or the cleaving method [32]. In the only colloid experiment to date, Hernández Guzmán and Weeks obtained $\tilde{\gamma}=(1.20 \pm 0.05) k_{B} T / \sigma^{2}$ [24]. The solid phase in that work consisted of a randomly stacked hexagonal crystal terminated by an interface with the fluid phase with ill-defined orientation. In the experiments presented in this paper we aimed for well defined interfaces along the high symmetry planes of the fcc crystals. We achieved this by preparing crystals from spherical silica particles that were deposited onto of lithographically prepared templates by sedimentation, a method pioneered by van Blaaderen et al. [33] that has been employed successfully to prepare high quality fcc crystals with dynamics similar to those of fcc atomic crystals $[34,35]$.

\section{EXPERIMENTAL DETAILS}

Spherical silica particles (micromod Sicastar ${ }^{\mathrm{TM}}$ plain) [45] with a diameter of $\sigma=1.55 \mu \mathrm{m}$ and a polydispersity of less than $3.5 \%$ [34] were suspended into a solution of $62.8 \mathrm{vol} \%$ dimethyl sulfoxide (DMSO) and $37.2 \%$ water that matched the particles' index of refraction. The solvent contained a small amount of fluorescein- $\mathrm{NaOH}$ for confocal fluorescence microscopy. The particles had a gravitational length of $k_{B} T / m^{*} g \approx \sigma / 7$ where $m^{*}$ is the relative mass and $g$ the gravitation constant. Starting from a homogeneous dispersion with a volume fraction of $10^{-4}$ the particles settled to the bottom of the cell and formed a crystalline sediment with a fluidlike layer on top. The growth of a single crystal with a particular orientation was directed by a template at the bottom of the cell [33]. A template consisted of a patterned $\sigma / 2$ thick poly(methyl methacrylate) film on a glass microscope cover slip. After fabricating the film by spin coating we used photolithography and reactive ion etching to produce a pattern of holes that resembled the first layer of the desired crystal orientation [46]. For thick crystals grown without templates we observed an interparticle distance of $a_{0}$ $=1.63 \mu \mathrm{m}$ at the bottom, and the template lattice parameters were chosen accordingly. The patterns covered a $5 \mathrm{~mm}$ quadratic area consisting of $3000^{2}$ primitive unit cells. Thus, the lateral size of the crystal exceeded that of the volume observed in the confocal microscope by two orders of magnitude.

The templates formed the base plate of a sample cell that we built from microscopy slides and glass glue. The cell was filled with suspension and left on the shelf until the particles had sedimented and formed a crystal of typically 15 layers. If a thicker crystal was desired, we replaced the liquid with fresh suspension and repeated the sedimentation step. For the experiments presented in this paper, we prepared typically 20-30 layers. Experiments were not started sooner than $12 \mathrm{~h}$ after the particle density profile had reached its equilibrium.

The sample was mounted on a Leica TCS SP5 point scanning confocal microscope and imaged from below through a $100 \times$ oil immersion objective using an excitation wavelength of $488 \mathrm{~nm}$. Confocal images consisted of 512 $\times 512$ pixel bitmaps (squares $70-100 \mu \mathrm{m}$ on a side), in which the nonfluorescent particles appeared dark in the dyed 

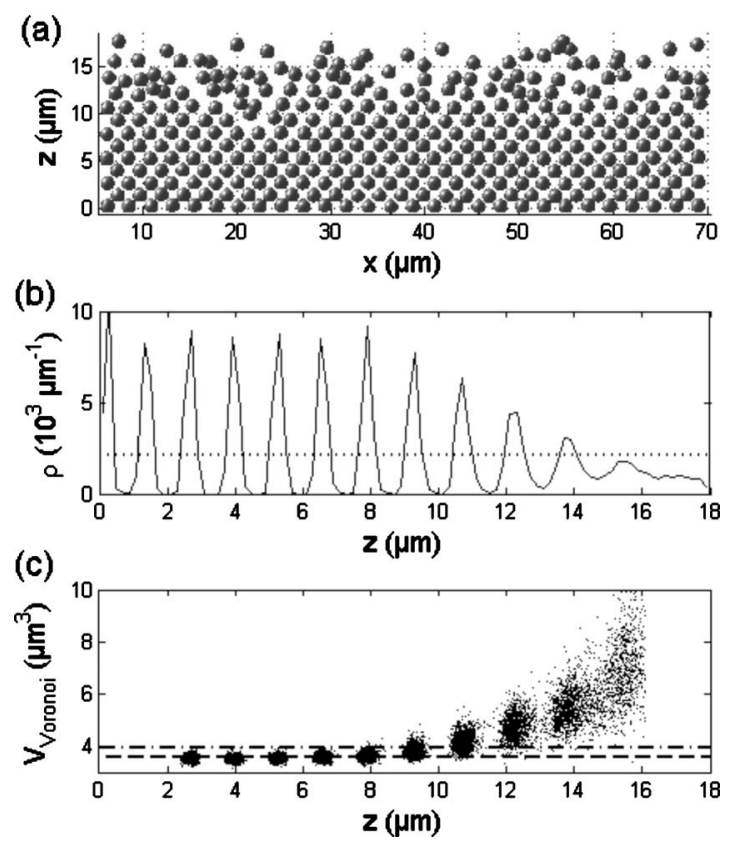

FIG. 1. (a) Graphic reconstruction of an $x-z$ slice from a (100) crystal-liquid interface. (b) Particle density profile of the same data set with the dotted line marking the overall average density. (c) Voronoi volumes as a function of $z$ with the two horizontal lines indicating the bulk crystalline (bottom) and liquid (top) volumes.

liquid. Scans in $z$ (vertical direction, step size typically $0.2-0.25 \mu \mathrm{m})$ provided image stacks with threedimensional voxel data. Measuring a full stack took typically $20-50 \mathrm{~s}$, depending on the number of $z$ steps and scanning parameters.

The raw data were processed using an IDL software package [36] based on algorithms by Crocker and Grier [37]. Noise and spatial intensity variations are removed by a bandpass filter. The image quality is sufficient to pinpoint the $(x, y, z)$ positions of the stationary particles with an accuracy of $0.1 \sigma$.

\section{RESULTS AND DISCUSSION}

A crystal was grown onto a template that consisted of square $a_{0} \times a_{0}$ unit cells, which corresponds to the (100) plane of an fcc lattice with a lattice parameter of $a_{0} \sqrt{(2)}$. Figure 1(a) shows a vertical $x-z$ slice that intersects the interface plane. One can clearly distinguish the single crystal from the disordered region on top. The particles in the disordered layer are moving and are not arrested in a glassy state. As a first step toward characterizing the structure we calculated the particle density profile

$$
\rho(z)=\frac{1}{\Delta z} \iiint_{z}^{z+\Delta z} d x d y d z \rho(x, y, z)
$$

for the entire sampled volume by integrating the particle density $\rho(x, y, z)$ over $\Delta z=0.2 \mu \mathrm{m}$ thick $x-y$ slabs. For small $z$ the profile shown in Fig. 1(b) exhibits the typical signature of a crystal: pronounced peaks with $\rho(z)$ dropping to zero inbetween. For larger $z$, these peaks broaden into oscillations with a decaying envelope. The transition is discontinuous. The dotted line indicates the particle density averaged over the entire $z$ range. Figure 1(c) shows the Voronoi volumes for some particles as a function of their $z$ positions [47]. The horizontal lines in the diagram mark the gap between the crystalline and fluid phase in the hard sphere phase diagram (3.58 and $3.95 \mu \mathrm{m}^{3}$, corresponding to volume fractions 0.545 and 0.494 , respectively) [27]. As expected, the Voronoi volumes in the apparently crystalline region lie within a narrow distribution below that gap, while the particles in the top region are associated with a broader range of much larger Voronoi volumes. All three subsets of the figure give a clear indication that our system consists of two phases (crystalline and fluidlike) separated by a horizontal average interface. Figure 1(a) suggests a corrugated, but locally sharp interface, not a gradual one with a smooth density profile. This will now be demonstrated quantitatively.

To distinguish the crystalline from the fluid phase we assign an order parameter to each individual particle based on the arrangement of the neighboring particles. Several simulation studies compare for this purpose the local structure to an idealized one [6,38]. Another well established method is the use of bond orientation order parameters $[39,40]$. For a number of reasons we favor a simplified version of the approach introduced by Ackland and Jones [41]. If $r_{i j}$ is the distance between a particle $i$ and any other particle $j$, one can identify the six particles closest to $i$ and calculate their mean squared separation

$$
r_{0}^{2}=\frac{1}{6} \sum_{j=1}^{6} r_{i j}^{2} .
$$

All particles with $r_{i j}^{2}<1.45 r_{0}^{2}$ are considered near neighbors of $i$. Next, the bond angles $\theta_{j i k}$ between any two neighbor pairs are calculated. The order parameter $\phi_{i}$ is the number of angles with $\cos \left(\theta_{j i k}\right)=0.5 \pm 0.2\left(\theta_{j i k} \approx 60^{\circ}\right)$. In an ideal closed packed crystal (face centered cubic or hexagonal close packed) it is 24-the highest possible value for the order parameter. Vacancies, interstitials and other point defects in the crystal lower the order parameters in their vicinity. Since this would complicate the identification of the interface later on, the order parameters are averaged by calculating the mean value for every particle $i$ and its neighbors, using the same $<1.45 r_{0}^{2}$ neighbor criterion as before. The local average $\langle\phi\rangle_{i}$ is then assigned to the central particle $i$.

The order parameters obtained in this way are visualized in Fig. 2(a). It shows a graphic reconstruction of the particle positions for part of the data of Fig. 1(a). Figure 2(b) shows the order parameters for the complete data set as a function of $z$. Despite the use of different order parameters, the graph agrees qualitatively with similar ones shown in simulation studies [29]. Comparison of Figs. 2(a) and 2(b) reveal that the width of the transition in $z$ does not represent the intrinsic width of the interface. Instead, it is the combined result of intrinsic width and interface corrugation. Both diagrams confirm that $\langle\phi\rangle_{i}$ is a good identifier of the two phases. It is instructive to quantify the relative occurrence of the various order parameter values, as visualized in the histogram of Fig. 2(c). The bar to the far right represents average order param- 
(a)

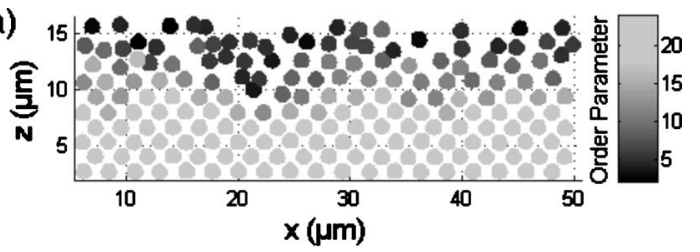

(b)

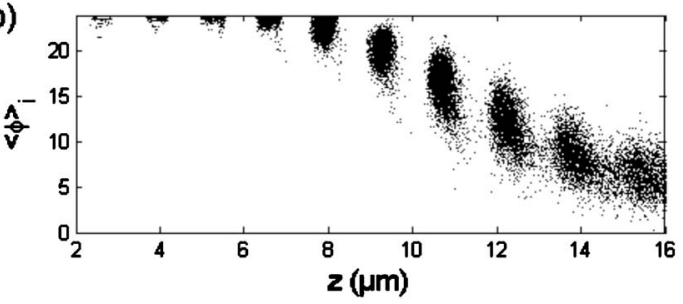

(c)

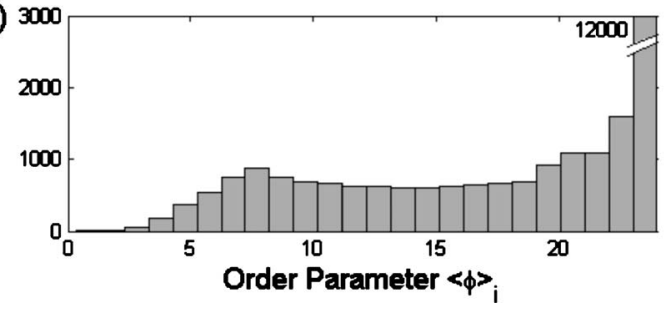

FIG. 2. (a) Graphic reconstruction of the same data set as in Fig. 1 with the locally averaged order parameter $\langle\phi\rangle_{i}$ for each particle labeled in grayscale. (b) $\langle\phi\rangle_{i}$ as a function of $z$. (c) Total occurrence of the individual $\langle\phi\rangle_{i}$ within the data set.

eters within the interval $23<\langle\phi\rangle_{i}<24$ and thereby most particles of the bulk crystalline phase. Smaller order parameters occur less frequently, but there is an apparent secondary maximum around $6<\langle\phi\rangle_{i}<9$. We take this as an indication that there is indeed a true, although thin, fluid phase and that we do not observe merely a gradual transition from a colloidal crystal to the empty solvent above. The histogram suggests that intermediate order parameters $\left(13<\langle\phi\rangle_{i}<16\right)$ are characteristic for particles at the interface and could be used to locate it. However, point defects within the crystal or coincidental configurations within the fluid phase can also cause order parameters in that numerical range and any automated analysis would need an elaborate identification and discrimination of these occurrences. A more elegant approach to solve this problem has been introduced by $\mathrm{Mu}$ et $a l$. [29]: first one defines a threshold value $\bar{\phi}_{s}$ and considers all particles with $\langle\phi\rangle_{i} \geq \bar{\phi}_{s}$ as part of the crystalline (solid) phase. Next, for every "solid" particle identified in this way the number $Z_{i}^{n n s}$ of solid neighbors fulfilling the same criterion is calculated. Within the bulk of a perfect fcc crystal $Z_{i}^{n n s}=12$. In order to identify the crystal-melt interface, one has to look out for "solid" particles with $\langle\phi\rangle_{i} \geq \bar{\phi}_{s}$, but only few solid neighbors $\left(Z_{i}^{n n s}<Z_{s}\right)$, introducing a second threshold parameter $Z_{s}$. The result of this selection scheme depends on the choice of the two parameters $\bar{\phi}_{s}$ and $Z_{s}$. Obviously, the number of identified interface particles increases with $Z_{s}$ in a strictly monotonic way. If one chooses $Z_{s}$ too small, only very few particles are identified. The result is a patchy and (if interpolated) deceptively smooth interface. Too high a $Z_{s}$, on the other hand, yields too many particles, which complicates the exact localization of an interface profile. With respect to
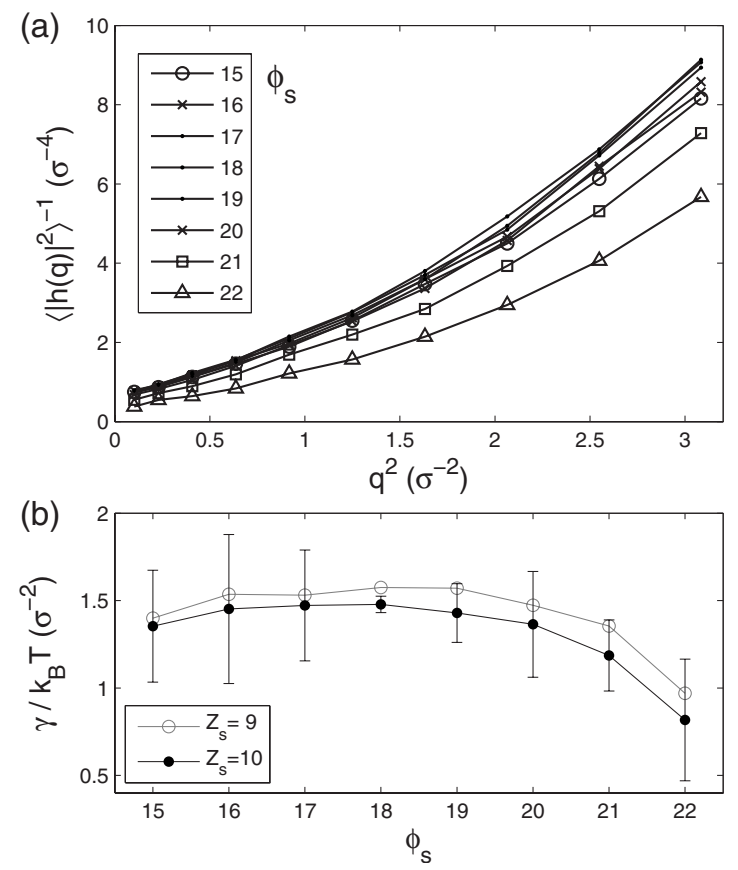

FIG. 3. (a) Inverse of the averaged squared Fourier components of the interfacial height profile vs $q^{2}$ for $Z_{s}=10$ and $15 \leq \bar{\phi}_{s} \leq 22$ and (b) the interfacial stiffness $\tilde{\gamma}$ obtained from fitting the linear sections of the curves (error bars from fit, omitted for $Z_{s}=9$ ).

the choice of $\bar{\phi}_{s}$ we found that it can shift the interface in $z$ by one or two layers while maintaining its general structure. Extreme values $\left(\bar{\phi}_{s}>22\right)$ tend to yield too many interface particles as well. Considering that the square conventional (100) surface of a perfect fcc crystal has two particles per unit cell, we choose $Z_{s}$ so as to obtain interfaces with an area density between 1 and 2 particles per unit cell area. This restricts our choice to $Z_{s}=9$ or $Z_{s}=10$ for $7 \leq \bar{\phi}_{s} \leq 22$.

Mu et al. showed that the final result (the stiffness of the interface obtained via CFM) seems rather robust with respect to the choice of $Z_{s}$, but depends to a greater degree on $\bar{\phi}_{s}$ [29]. One has to be careful when applying these insights directly to our work, since the earlier work uses not only a different order parameter (not practical for our data), but also a quasi-two-dimensional system (periodic boundaries with 4 crystal layers in the narrow direction). For reasons given above, we have already narrowed down our choice of $Z_{s}$ to 9 or 10 . To justify the choice of the value of $\bar{\phi}_{s}$ we have performed for each integer value $15 \leq \bar{\phi}_{s} \leq 22$ the complete data evaluation (explained later on) to a data set of 120 interface configurations. The results are summarized in Fig. 3: Plot (a) shows the inverse averaged squared Fourier components of the height profiles versus $q^{2}$ for all $\bar{\phi}_{s}$ considered. According to Eq. (2) we expect a linear relationship in the long wavelength limit, which is more or less fulfilled for all curves. The nonlinear behavior at higher $q^{2}$ values will be discussed later on, but even in that region the choice of $\bar{\phi}_{s}$ has only minor effect on the Fourier components: Except for the extreme cases $\left(\bar{\phi}_{s}>20\right)$ all curves lie very close together, aim for a mutual intersection point with the vertical axis, and approach it at similar slopes. This demonstrates the robust- 
ness of our algorithm. The interfacial stiffness so calculated from a linear fit to the first four points of each curve, give the relation between $\bar{\phi}_{s}$ and $\tilde{\gamma}$ shown in Fig. 3(b), which is qualitatively similar to the results of the simulation study cited above [29]. It has been argued in that work that if $\bar{\phi}_{s}$ is very large or very small, additional particles from the fluid or solid phase are wrongly identified as interface particles, which artificially increases the interface roughness and in turn leads to an underestimation of the interfacial stiffness $\tilde{\gamma}$. The authors argue further that the value of $\bar{\phi}_{s}$ for which one obtains the highest $\tilde{\gamma}$ reveals the true interface and therefore the correct $\tilde{\gamma}$. In our case we observe a broad maximum around $\bar{\phi}_{s} \sim 18$. We repeated the procedure for $Z_{s}=9$ with a similar result, as shown in Fig. 3(b). In the following we use $Z_{s}=10$ and $\bar{\phi}_{s}=18$.

Randomly picked examples for interface configurations obtained in this fashion consisted of particle densities that were homogeneous in $x$ and $y$ with well defined interfacial dividing surfaces. Ambiguities caused by two or more particles on top of each other or by large "holes" in the interface layer were extremely rare. From the $(x, y, z)$ coordinates of the particles we obtained by interpolation a height function $h(j, k)$ on a $\Delta_{x y}=0.5 \mu \mathrm{m}$ spaced $L \times L$ quadratic grid aligned with the symmetry axes of the crystal. The reference level $(h=0)$ of the height profile is the mean $z$ value. In order to reduce truncation artifacts in the Fourier transform, $h(x, y)$ was gradually brought to zero at the edges. In practice this was achieved by multiplying the data within a $5 \%$ border with a quarter-period sine function. The Fourier components $h(m, n)$ on the corresponding reciprocal grid were obtained according to

$$
h(m, n)=\frac{\Delta_{x y}^{2}}{\sqrt{A}}\left[\sum_{j k} h(j, k) e^{-i(2 \pi / L) m j} e^{-i(2 \pi / L) n k}\right]
$$

with $A$ the total area in real space and $L$ the number of grid points.

We measured 180 configurations of the (100) interface within a $69 \times 69 \times 20 \mu^{3}$ volume sampled at 1 min intervals $(3 \mathrm{~h}$ in total) and 120 configurations sampled at $5 \mathrm{~min}$ intervals $(10 \mathrm{~h})$ under otherwise identical conditions. Analyzing both data sets separately we found qualitatively identical results with minor quantitative discrepancies as discussed below. Therefore, for the sake of better statistics, we treated the measurements as one data set with 300 configurations. The resulting inversed averaged squared Fourier component are shown in Fig. 4(a) as a grayscale map. For better image quality, we exploited the four mirror symmetries of the (100) interface, which restricted the independent data points to the triangular zone marked in the figure.

The pattern appears to be azimuthally symmetric at the center. That suggests that the mean square amplitude $\left\langle|h(\mathbf{q})|^{2}\right\rangle^{-1}$ of a long wavelength capillary fluctuation mode is independent of the direction of its wave vector $\mathbf{q}$ within the (100) interface plane. Since Eq. (2) links the ratio between $\left\langle|h(\mathbf{q})|^{2}\right\rangle^{-1}$ and $q^{2}$ (at least in the long wavelength limit) directly to the interfacial stiffness, the result indicates the isotropy of the interface stiffness in (100). In other words, the

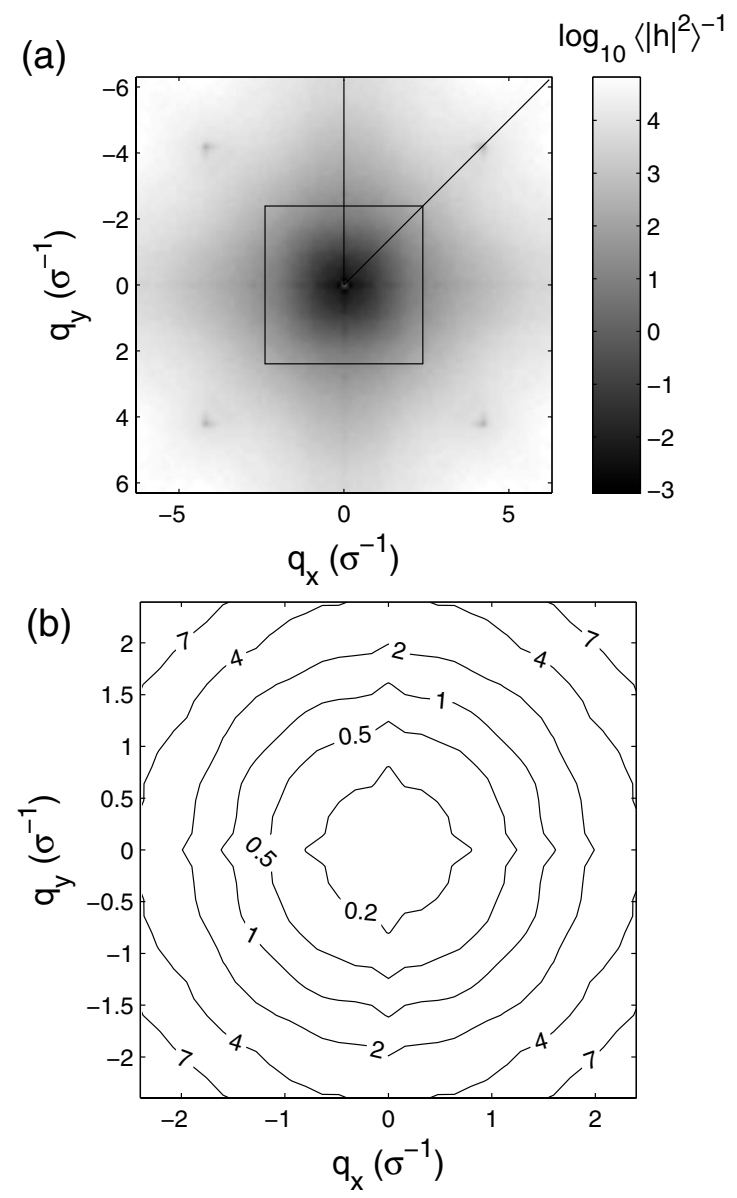

FIG. 4. (a) Logarithmic grayscale plot of the inverse averaged squared Fourier components of the (100) interfacial height profile. Noise was reduced by symmetry averaging (the triangle indicates one symmetry unit). The square marks the region shown as linear contour plot in (b).

resistance of the interface against curvature is independent of the direction about which the interface is curved. This is exactly the behavior expected from the interface stiffness tensor of a cubic crystal [42]. For high $q$ values the associated wavelengths approach the particle scale and we observe anisotropies. However, in order to show the azimuthal symmetry for small $q$ even more clearly, Fig. 4(b) displays the center region of Fig. 4(a) as a contour plot.

In order to increase the statistical quality further, we calculated the Fourier components as a function of $|q|$ by azimuthally averaging the data of Fig. 4, and plotted the result vs $q^{2}$ in Fig. 5. In agreement with previous work we observe a deviation from the linear behavior for shorter wavelengths. This is to be expected, since a continuum approach such as CFM has to break down when the length scale approaches that of the particles. The fit to the linear section (the first four data points) has a slope of $\tilde{\gamma}=(1.3 \pm 0.3) k_{B} T \sigma^{-2}$ and an offset of $\alpha=(0.57 \pm 0.10) \sigma^{-4}$. Both data sets evaluated separately yielded within the error the same results, with a particularly good agreement in the linear, long-wavelength region. We repeated the experiment under different conditions (fresh sample, different microscope, $94 \times 94 \times 15 \mu \mathrm{m}^{3}$ volume sampled 70 times at 2 min intervals) and obtained $\tilde{\gamma}$ 


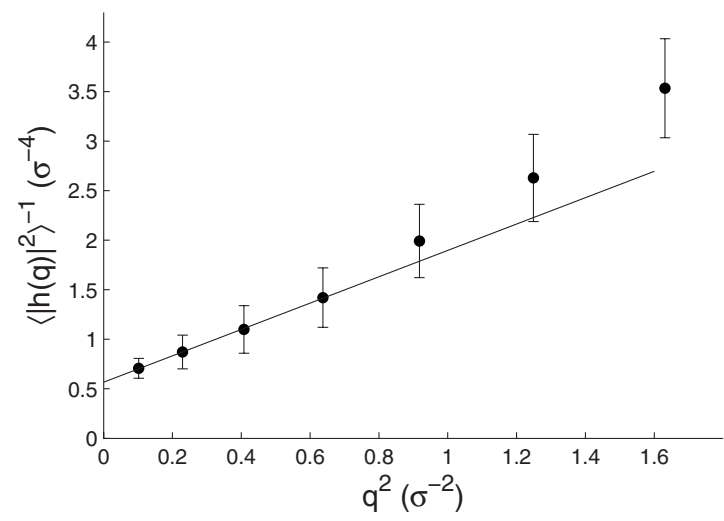

FIG. 5. Inverse of the averaged squared Fourier components of the (100) interfacial height profile vs $q^{2}$ with a linear fit to the long wavelength $\left(q^{2}<0.8 \sigma^{-2}\right)$ section.

$=1.1 k_{B} T \sigma^{-2}$ and $\alpha=0.49 \sigma^{-4}$. The error bars in Fig. 5 and the uncertainties given in the numerical results reflect the scatter between the individual data sets. The uncertainty in the slope and therefore $\tilde{\gamma}$ is due to these errors and to the somewhat $a d$ hoc definition of the long wavelength region.

Although our result is subject to substantial uncertainty, the stiffness we obtain is nonetheless clearly about twice as high as those reported in simulations. A simple explanation for this discrepancy could be an imperfect realization of the hard sphere interaction potential in our system. However, the only other experimental study known to us obtains essentially the same result $\left(\tilde{\gamma}=1.2 k_{B} T \sigma^{-2}\right)$ for a low-symmetry interface between a randomly stacked hexagonal closed packed (hcp) crystal and its melt [24].

That the plot of $\left\langle|h(\mathbf{q})|^{2}\right\rangle^{-1}$ vs $q^{2}$ does not intersect the coordinate axes at the origin but at an offset value $\alpha$, can be understood from the more general capillary wave theory, which takes into account the difference in mass density $\Delta \rho$ between the crystalline and fluid phases,

$$
\left\langle\left|h_{\mathbf{q}}\right|^{2}\right\rangle^{-1}=\frac{\tilde{\gamma}(\theta)}{k_{B} T}\left(q^{2}+\frac{g \Delta \rho}{\tilde{\gamma}(\theta)}\right),
$$

with the gravitational constant $g=9.81 \mathrm{~m} / \mathrm{s}^{2}$. This takes into account the additional damping of interface fluctuations by gravity [17]. From the measured offset $\alpha=0.57 \sigma^{-4}$ we calculate

$$
\Delta \rho=\alpha \frac{k_{B} T}{g}
$$

to be $41.7 \mathrm{mg} / \mathrm{cm}^{3}$. For the water/DMSO solution with a mass density of $\rho_{\text {solv }}=1.06 \mathrm{~g} / \mathrm{cm}^{3}$ and silica particles with $\rho_{\text {Silica }}=2 \mathrm{~g} / \mathrm{cm}^{3}$ this corresponds to a difference in volume fraction of 0.044 which reasonably agrees with the gap of 0.051 between the fluid $(\Phi \leq 0.494)$ and the crystal $(\Phi$ $\geq 0.545)$ phases in the hard sphere phase diagram $[27,28]$.

We performed a similar experiment using a $(110)_{f c c}$ template based on a rectangular $a_{0} \times \sqrt{2} a_{0}$ unit cell. 140 configurations were recorded at $5 \mathrm{~min}$ intervals amounting to a total time of almost $12 \mathrm{~h}$. The $\left\langle|h|^{2}\right\rangle^{-1}$ values, obtained by the same evaluation steps as in the (100) experiment, are pre-
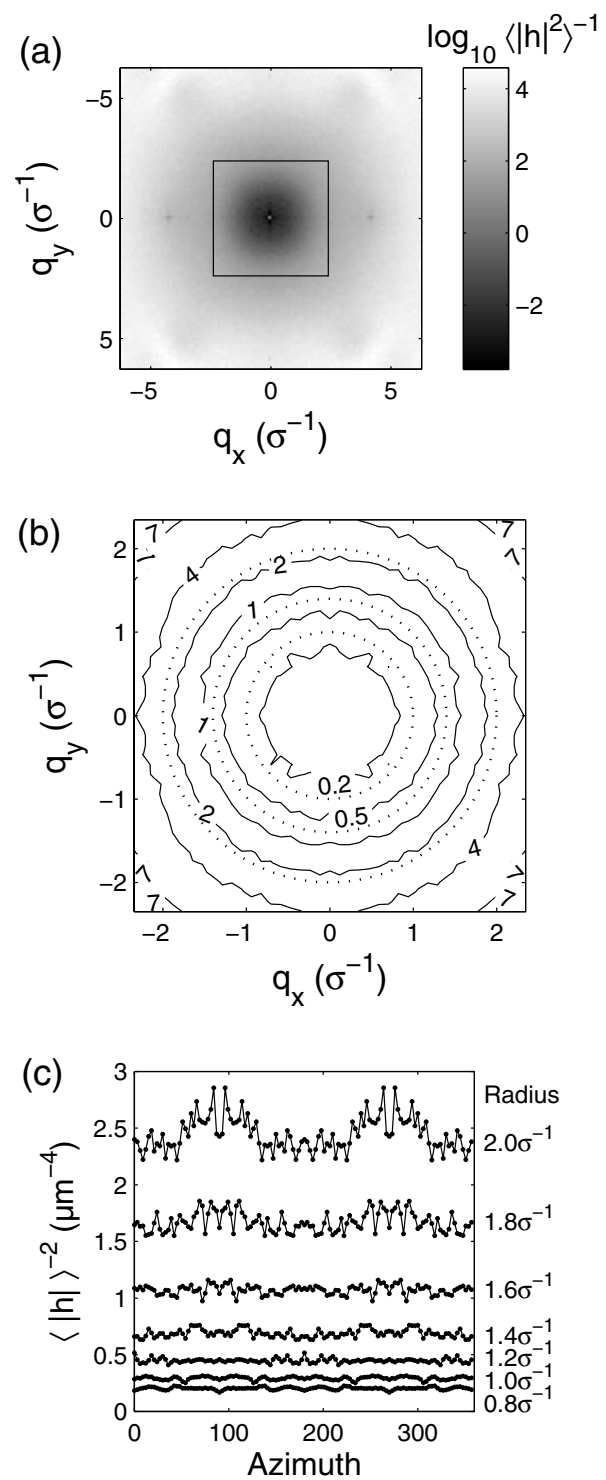

FIG. 6. (a) Logarithmic grayscale plot of the inverse averaged squared Fourier components of the (110) interfacial height profile. The square marks the region shown as linear contour plot in (b), with the dotted circles illustrating the azimuthal symmetry. (c) shows a series of circular scans at various radii.

sented in Fig. 6(a). The two (110) mirror planes were exploited to smooth the grayscale map. We observe the same azimuthal isotropy in the long wavelength limit [shown in detail by the contour plot Fig. 6(b)] as for the (100) data. The dotted circles in the figure are guides to the eye. Additionally, Fig. 6(c) shows circular data scans at various radii. The isotropy gives way to the twofold (110) rotational symmetry above $|q| \sim 1.8 \sigma^{-1}$. This value corresponds to a wavelength $2 \pi / q$ of less than four particle diameters, a length scale clearly beyond the scope of the CFM. In fact, the appearance of the twofold (110) symmetry pinpoints impressively the breakdown of the continuum approach.

The (110) long-wavelength isotropy comes as a surprise, since, different from (100) or (111), the (110) interface has no threefold or higher rotational symmetry that would induce this behavior [42]. In fact, significant differences in $\widetilde{\gamma}_{(110)[\overline{1} 10]}$ 


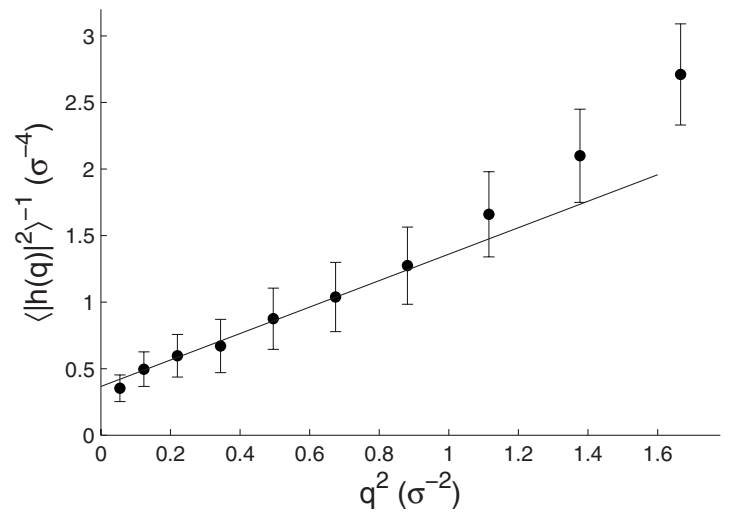

FIG. 7. Inverse of the averaged squared Fourier components of the (110) interfacial height profile vs $q^{2}$ with a linear fit to the long wavelength $\left(q^{2}<0.8 \sigma^{-2}\right)$ section.

and $\widetilde{\gamma}_{(110)[001]}$ have been observed in simulations (see Table I) $[7,29,30]$.

From a linear fit to the long wavelength section as shown in Fig. 7 we obtain $\widetilde{\gamma}_{110}=(1.0 \pm 0.2) k_{B} T \sigma^{-2}$ and an offset $\alpha_{110}=(0.37 \pm 0.10) \sigma^{-4}$. Error on the individual data points were estimated from the scatter of values obtained by analyzing only parts of the data.

Finally, we performed an experiment with a (111) template that consisted of a hexagonal pattern of holes with the lattice constant $a_{0}$. In principle we would not need a template to grow a crystal in this orientation, since it would form on top of a flat bottom wall as well. However, the template helps us to avoid grain boundaries. A fundamental difference to the previously described experiments is that growing a sequence of hexagonal closed packed layers of hard spheres results usually neither in an fcc crystal with its well known ABCABC type stacking sequence, nor in a hexagonal close packed (hcp) crystal with ABAB stacking. Although the equilibrium crystal structure of the hard sphere system is fcc, the free energy of a stacking fault is very small compared to $k_{B} T[43,44]$. Therefore, a randomly stacked hexagonal lattice occurs instead. For (100) and (110) layers the stacking sequence is unambiguous and good fcc crystals can be obtained.

That the (111) experiment was strictly speaking on a different crystal structure than the others was just one difficulty. For unknown reasons, the crystals obtained were not of the same quality as in the previous experiments. Major defect clusters persisted within the crystalline phase and some of them extended to the interface. Identifying the interface required fine tuning of the parameters: we got better results with $\bar{\phi}_{s}=15$ instead of 18 , which proved to be a too strict a criterion for crystallinity. The interface seemed more "fuzzy" than in the other cases, although this may be due to the defects.

As for the other orientations, we observe azimuthal symmetry in Fourier space as shown in Fig. 8. The linear fit to the long wavelength section shown in Fig. 9 gives $\tilde{\gamma}_{111}$ $=0.66 k_{B} T / \sigma^{2}$ and $\alpha_{111}=0.08 \sigma^{-4}$. These rather small values are quite unexpected, since the simulations report $\widetilde{\gamma}_{111}$ to be higher than $\widetilde{\gamma}_{(100),(110)}$ (see Table I). We attribute this discrepancy to the additional perturbation of the interface by the

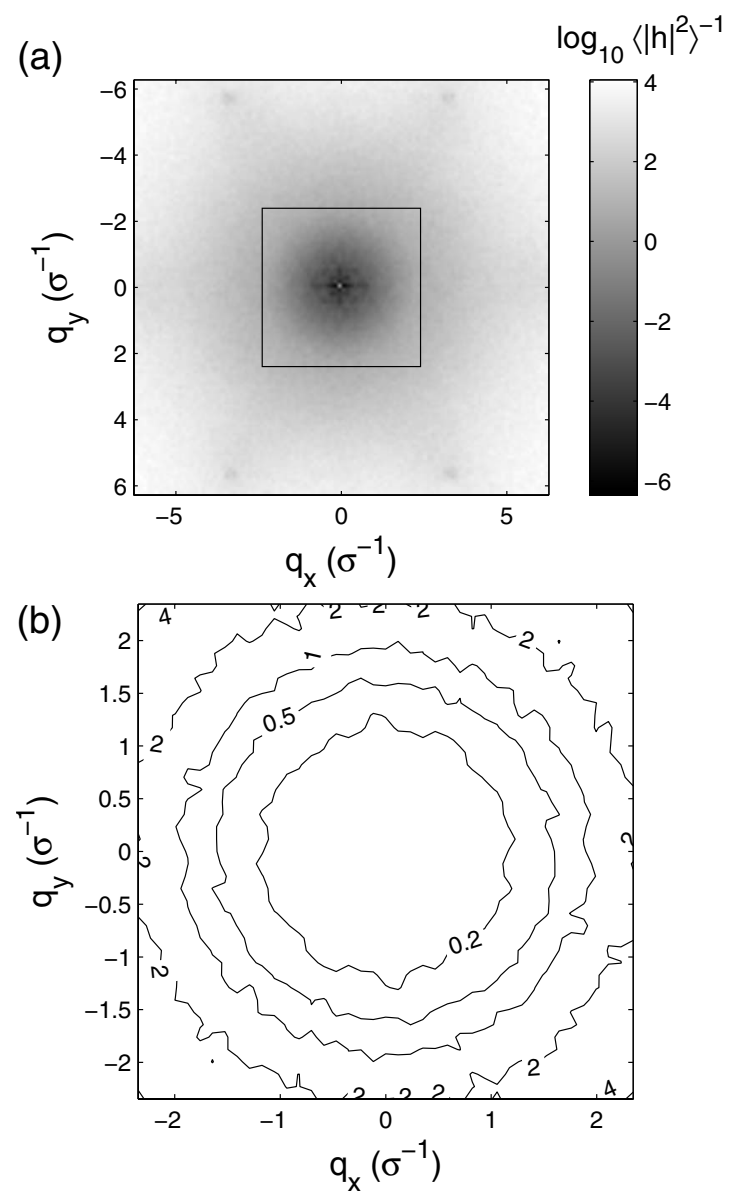

FIG. 8. (a) Logarithmic grayscale plot of the inverse averaged squared Fourier components of the interfacial height profile for an (111) interface. The square marks the region shown as linear contour plot in (b).

defects, which would naturally lead to an underestimation of the stiffness. The same may be true for the small $\alpha_{111}$, although it could also be that the defects reduce the packing fraction of the crystal and thereby the mass density difference between the crystalline and fluid phase. In general we consider the numerical results for this experiment unreliable and present this data set mainly to show the symmetry.

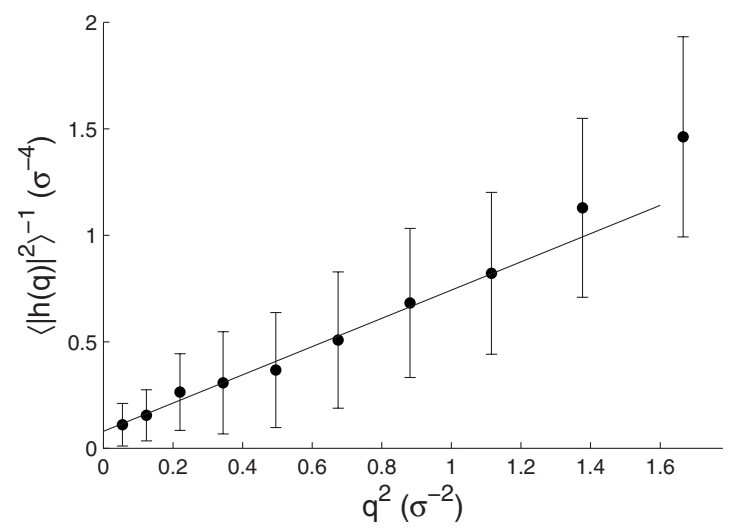

FIG. 9. Inverse of the averaged squared Fourier components of the (111) interfacial height profile vs $q^{2}$ with linear fit to the long wavelength section. 


\section{CONCLUSIONS}

We have used spherical silica colloids to realize experimentally a scenario that has been at the focus of considerable simulation efforts within recent years: the interface between a crystal and its own melt. Confocal microscopy enables us to study the colloidal model system at particle length scales, but at the same time over sufficiently large spatial dimensions to allow the application of continuum concepts such as the CFM. In close analogy to the simulation results in the literature, we determine the crystalline and fluid phases with single particle resolution and can pinpoint their interface, which is corrugated by capillary fluctuations. Our data allow a clear distinction between the sharp intrinsic interface and the macroscopic interfacial width which is due to said fluctuations. All this remains experimentally elusive for dynamic atomic systems. We have chosen quite heavy particles with a relatively small gravitational height of $\sigma / 7$ in order to obtain high quality crystals and a well defined horizontal interface. As a tradeoff, the fluid layer on top has a thickness of just a few particle diameters. In fact, the gravitational height is considerably smaller than the interface width. This probably introduces additional hard to quantify terms into Eq. (6) and affects the precision of our results. By directing crystal growth using microstructured templates we were able to prepare interfaces parallel to all three high-symmetry planes of the fcc crystal system: (100), (110), and (111). In all three cases we observe fluctuation amplitudes and a wavelength dependence in agreement with CFM. The amount of data is not sufficient yet to calculate the interfacial stiffness with an absolute accuracy that reveals the anisotropies seen in simulation results. Therefore, we observe, within the error, identical stiffnesses for (100) and (110). Even taking into account the limited accuracy they are somewhat larger than those found in simulations. However, they agree very well with the only other experimental results we are aware of [24].

For all three high-symmetry orientations of the interface we observe azimuthal symmetry in the fluctuation wave modes of any data set: in the long wavelength limit the Fourier amplitudes seem to be entirely independent of the inplane direction of the associated wave vectors. While this perfectly agrees with theory for (100) and (111) interfaces, it comes as a surprise for the (110) interface. Here we would expect at least a relative difference, which would be consistent with the stiffness tensor of a cubic crystal, and has in fact been observed in earlier simulations.

We consider it exciting that there may be a systematic difference between experimental and numerical results and expect that resolving this discrepancy will be a challenging problem. Exciting and challenging, because both approaches have inherent and complementary strengths and weaknesses. The limited accuracy of experimental studies could be improved by more efficient data acquisition. It would also be worthwhile to repeat the experiments with smaller particles of lesser specific weight to investigate the possible influence of our relatively thin fluid layer and the consequences of the small gravitational height. It is important to keep in mind, however, that the limitations on system size and observation time due to scanning area and storage space do only concern the probed volume, not the sample, which is laterally larger by an order of magnitude and not a priori limited in size. The weakness compared to numerical studies is that colloidal systems can only approximate hard spheres to a finite degree. Residual interactions, gravity effects, and polydispersity can be minimized, but never removed altogether. Simulations, on the other hand, are limited in size and subject to periodic boundary conditions. Despite the importance of the hard sphere system, simulations will never model "reality" in every tiny aspect. However, a system that does not exist in "reality" will never be fully realized experimentally either. Resolving the issue presented here should be of interest to both communities and, in the end, improve the understanding of the underlying physical problems.

\section{ACKNOWLEDGMENTS}

We acknowledge helpful discussions with M. Asta and P. Schall. Valuable support was given by the staff of the Center of Nanoscale Systems (CNS) at Harvard, in particular by Y. Lu. This work was funded by NSF (Grant No. DMR0602684) and the Harvard MRSEC (Grant No. DMR0213805).
[1] J. Warren, Nature Mater. 5, 595 (2006).

[2] T. Haxhimali, A. Karma, F. Gonzales, and M. Rappaz, Nature Mater. 5, 660 (2006).

[3] J. J. Hoyt, M. Asta, and A. Karma, Mater. Sci. Eng. R. 41, 121 (2003).

[4] J. Broughton and G. Gilmer, J. Chem. Phys. 84, 5759 (1986).

[5] A. Karma, Phys. Rev. E 48, 3441 (1993).

[6] J. J. Hoyt, M. Asta, and A. Karma, Phys. Rev. Lett. 86, 5530 (2001).

[7] R. Davidchack, J. Morris, and B. Laird, J. Chem. Phys. 125, 094710 (2006).

[8] R. E. Napolitano, S. Liu, and R. Trivedi, Interface Sci. 10, 217 (2002).

[9] R. E. Napolitano and S. Liu, Phys. Rev. B 70, 214103 (2004).
[10] H. Yoshida, K. Ito, and N. Ise, Phys. Rev. B 44, 435 (1991).

[11] A. van Blaaderen and P. Wiltzius, Science 270, 1177 (1995).

[12] W. Russel, D. Saville, and W. Schowalter, Colloidal Dispersions (Cambridge University Press, Cambridge, England, 1989).

[13] R. Jones, Soft Condensed Matter (Oxford University Press, New York, 2002).

[14] G. Gompper and M. Schick, Colloidal Order: Entropic and Surface Forces, Soft Matter Vol. 3 (Wiley, New York, 2007).

[15] V. Prasad, D. Semwogerere, and E. Weeks, J. Phys.: Condens. Matter 19, 113102 (2007).

[16] D. G. A. L. Aarts, M. Schmidt, and H. N. W. Lekkerkerker, Science 304, 847 (2004).

[17] D. Aarts, M. Schmidt, H. Lekkerkerker, and K. Mecke, Adv. 
Solid State Phys. 45, 15 (2005).

[18] D. Derks, D. G. A. L. Aarts, D. Bonn, H. N. W. Lekkerkerker, and A. Imhof, Phys. Rev. Lett. 97, 038301 (2006).

[19] T. Biben, R. Ohnesorge, and H. Löwen, EPL 28, 665 (1994).

[20] H. Chen and H. Ma, J. Chem. Phys. 125, 024510 (2006).

[21] M. Marechal and M. Dijkstra, Phys. Rev. E 75, 061404 (2007).

[22] I. B. Ramsteiner, K. E. Jensen, D. A. Weitz, and F. Spaepen, Phys. Rev. E 79, 011403 (2009).

[23] R. P. A. Dullens, D. G. A. L. Aarts, and W. K. Kegel, Phys. Rev. Lett. 97, 228301 (2006).

[24] J. Hernández-Guzmán and E. Weeks, Proc. Natl. Acad. Sci. U.S.A. 106, 15198 (2009).

[25] W. Wood and J. Jacobson, J. Chem. Phys. 27, 1207 (1957).

[26] B. Alder and T. Wainwright, J. Chem. Phys. 27, 1208 (1957).

[27] W. Hoover and F. Ree, J. Chem. Phys. 49, 3609 (1968).

[28] P. Pusey and W. van Megen, Nature (London) 320, 340 (1986).

[29] Y. Mu, A. Houk, and X. Song, J. Phys. Chem. B 109, 6500 (2005)

[30] M. Amini and B. B. Laird, Phys. Rev. Lett. 97, 216102 (2006).

[31] D. Marr and A. Gast, Langmuir 10, 1348 (1994).

[32] R. L. Davidchack and B. B. Laird, Phys. Rev. Lett. 85, 4751 (2000).

[33] A. van Blaaderen, R. Ruel, and P. Wiltzius, Nature (London) 385, 321 (1997).
[34] P. Schall, I. Cohen, D. Weitz, and F. Spaepen, Science 305, 1944 (2004).

[35] P. Schall, I. Cohen, D. Weitz, and F. Spaepen, Nature (London) 440, 319 (2006).

[36] http://www.physics.emory.edu/ weeks/idl/

[37] J. Crocker and D. Grier, J. Colloid Interface Sci. 179, 298 (1996).

[38] J. R. Morris, Phys. Rev. B 66, 144104 (2002).

[39] P. J. Steinhardt, D. R. Nelson, and M. Ronchetti, Phys. Rev. B 28, 784 (1983).

[40] J. Honeycutt and H. Andersen, J. Phys. Chem. 91, 4950 (1987).

[41] G. J. Ackland and A. P. Jones, Phys. Rev. B 73, 054104 (2006).

[42] D. Du, H. Zhang, and D. Srolovitz, Acta Mater. 55, 467 (2007).

[43] L. Woodcock, Nature (London) 385, 141 (1997).

[44] P. Bolhuis, D. Frenkel, S.-C. Mau, and D. Huse, Nature (London) 388, 235 (1997).

[45] micromod GmbH, Rostock-Warnemuende, Germany, www.micromod.de.

[46] We used standard photolithographic processes. Lithography masks were produced with a DWL-66 Heidelberg mask writer.

[47] Voronoi volumes were calculated with MatLab functions based on QHull (www.qhull.org). 